\title{
$\beta$-TrCP-mediated ubiquitination and degradation of Dlg5 regulates hepatocellular carcinoma cell proliferation
}

\author{
Dongping Wang ${ }^{1 \dagger}$, Qi Zhang ${ }^{2+}$, Fenfen $\mathrm{Li}^{3+}$, Chan Wang ${ }^{1}$, Changming Yang ${ }^{1 *}$ (D) and Hong Yu ${ }^{4^{*}}$
}

\begin{abstract}
Background: Discs large homolog 5 (Dlg5) is a member of the membrane-associated guanylate kinase (MAGUK) adaptor family of proteins and its deregulation has been implicated in the malignancy of several cancer types. Dlg5 was down-regulated in hepatocellular carcinoma (HCC) and lower Dlg5 expression was associated with poor survival of HCC patients. However, how to regulate Dlg5 remains largely unknown.

Methods: The co-immunoprecipitation assay was used to determine the interaction between Dlg5 and $\beta$-TrCP. The in vivo ubiquitination assay was performed to determine the regulation of Dlg 5 by $\beta$-TrCP. CCK- 8 and colony formation assay were implemented to detect the biological effect of Dlg5 on the growth of HCC cells in vitro. The effect of Dlg5 on HCC tumor growth in vivo was studied in a tumor xenograft model in mice.

Results: Here we report that Dlg5 is regulated by the ubiquitin proteasome system and depletion of either Cullin 1 or $\beta$-TrCP led to increased levels of Dlg5. $\beta$-TrCP regulated Dlg5 protein stability by targeting it for ubiquitination and subsequent destruction in a phosphorylation-dependent manner. We further demonstrated a crucial role of Ser730 in the non-canonical phosphodegron of Dlg5 in governing $\beta$-TrCP-mediated Dlg5 degradation. Importantly, failure to degrade Dlg5 significantly inhibited HCC cells proliferation both in vitro and in vivo.

Conclusion: Collectively, our finding provides a novel molecular mechanism for the negative regulation of Dlg5 by $\beta$-TRCP in HCC cells. It further suggests that preventing Dlg5 degradation could be a possible novel strategy for clinical treatment of HCC.
\end{abstract}

Keywords: Dlg5, $\beta-\operatorname{TrCP}$, Wnt/ $\beta$-catenin signaling, HCC

\section{Background}

Liver cancer is the sixth most common cancer in the world and the second malignant tumor of global cancer mortality. It is composed of hepatocellular carcinoma (HCC), intrahepatic cholangiocarcinoma and mixed liver cancer, and about $90 \%$ is hepatocellular carcinoma [1]. The pathogenesis of hepatocellular carcinoma (HCC) is

*Correspondence: hbjmyangcm@163.com; 919336324@qq.com

${ }^{\dagger}$ Dongping Wang, Qi Zhang and Fenfen Li contributed equally to this work

${ }^{1}$ Department of Anesthesiology, The First People's of Hospital of Jingmen

City, Jingmen, Hubei 448000, China

${ }^{4}$ Department of Neonatology, The First People's of Hospital of Jingmen

City, Jingmen, Hubei 448000, China

Full list of author information is available at the end of the article extremely complicate, which is the result of multi-factors, multi-genes and multi-stages participation [2, 3]. Thus, the discovery of new molecular drug targets will benefit HCC treatment.

The ubiquitin proteasome pathway (UPP) is critical for protein degradation in eukaryotic organisms. It can selectively degrade many biologically active proteins in cells and participate in the regulation of almost all biological processes, including cell cycle, apoptosis and inflammatory response [4]. Ubiquitination requires the reaction of three enzymes including ubiquitin-activating enzyme $\mathrm{E} 1$, ubiquitin-binding enzyme E2, and ubiquitin ligase E3 [5]. E3 ligase plays the most critical role in the entire ubiquitination process by recognizing distinct substrates [6]. The F-box protein family is an important component 
of the evolutionarily conserved ubiquitin protein ligase complex SCF (Skp1/Cullin1/F-box protein). F-box proteins are able to recruit substrates and promote their ubiquitination and degradation. The F-box protein family has many members, and nearly 70 F-box protein members have been reported in humans [7, 8]. They are classified into three broad categories based on their domain characteristics: the FBXW family with WD40 domain, the FBXL family with leucine-rich domain, and the FBXO family with other domains [9]. However, only a few of these SCF ubiquitin ligases have identified substrates. Noticeably, phosphorylation on specific sites are requested for most SCF substrates which are recognized by most $\mathrm{F}$ box proteins [10].

Discs large homolog 5 (Dlg5) is a member of the membrane-associated guanylate kinase (MAGUK) adaptor family of proteins [11]. The down-regulation of Dlg5 has been implicated in the malignancy of HCC [12]. However, how to regulate Dlg5 in HCC is largely unknown. Here we show that the F-box protein $\beta$-TrCP regulates Dlg5 protein stability by targeting it for ubiquitination and subsequent destruction in a phosphorylationdependent manner. The Ser730 phosphorylation of Dlg5 is critical for $\beta$-TrCP-mediated Dlg5 degradation, and failure to degrade Dlg5 significantly inhibits HCC cells proliferation both in vitro and in vivo.

\section{Materials and methods Cell culture and reagents}

HEK293T cells and hepatocellular carcinoma cell lines SMMC-7721 and HepG2 cells were purchased from Cell Bank of Shanghai Institute of Biological Science (SIBS, CAS, Shanghai, China). Cells were cultured in Dulbecco's modified Eagle's medium (DMEM) (Invitrogen), supplemented with $10 \%$ FBS (Gibco), 100 units/mL penicillin, and $100 \mathrm{mg} / \mathrm{mL}$ streptomycin (Gibco). MLN4924 was purchased from Medkoo. Cycloheximide (CHX) was purchased from Sigma.

\section{Plasmids}

Dlg5, $\beta$-TrCP and ubiquitin were amplified from SMMC7721 cells by polymerase chain reaction and cloned into pcDNA3.1 or pbabe-Flag vector. Five pcDNA3DN-hCULs-FLAG were gifts from Wade Harper (Addgene plasmid \#15818; \#15819; \#15820; \#15821 and \# 15822). Dlg5 mutant were generated using QuickChange Site-Directed Mutagenesis Kit (Stratagene). pRK5-HA-Ubiquitin-K48 was a gift from Ted Dawson (Addgene plasmid \# 17605; http://n2t.net/addgene:17605 ; RRID:Addgene_17605). Ub-K48 was then cloned into pcDNA3-his vector. All cDNAs were completely sequenced. All plasmids were transfected into cells using
Lipofectamine 2000 (Invitrogen) according to manufacturer instructions.

\section{RNA interference}

All siRNAs were transfected into cells at $100 \mathrm{nM}$ using Lipofectamine RNAimax (Invitrogen). The sequences of $\beta$-Trcp siRNA: 5 -AAGUGGAAUUUGUGGAACAUC$3^{\prime}$. The siRNA targeting Cullin1 were purchased from Santa Cruz with cat \# sc-35126.

\section{Construction of stable cell line}

Viral supernatants were produced in HEK293T cells co-transfected with the pBabe-Flag-control, pBabeFlag-Dlg5 WT or pBabe-Flag-Dlg5 S730A plasmids and packaging vectors. Viral supernatants were collected 48 and $72 \mathrm{~h}$ after transfection. Filter-sterilized viral supernatants were added to the cells with $10 \mu \mathrm{g} / \mathrm{mL}$ Ploybrane for $48 \mathrm{~h}$ and selected with puromycin $(2 \mu \mathrm{g} / \mathrm{mL})$ for a week.

\section{Western blotting and antibodies}

Cells were lysed in SDS loading buffer. The boiled samples were separated by $10 \%$ SDS-PAGE and transferred to nitrocellulose (NC) membranes (Whatman, GE Healthcare). The membranes were blocked with 5\% milk and incubated with different antibodies overnight at 4 , followed by incubation with secondary antibodies. The primary antibodies used in western blotting included anti-Flag M2, anti-HA, anti-Ub, anti-Dlg5 (Sigmaaldrich); anti- $\beta$-TrCP (Cell signaling) and anti-Cullin1, anti-SKP1 and anti- $\beta$-actin (Santa Cruz).

\section{Immunoprecipitation (IP)}

Cells were lysed in lysis buffer $(50 \mathrm{mM}$ Tris- $\mathrm{HCl} \mathrm{pH} 7.5$, $150 \mathrm{mM} \mathrm{NaCl}, 0.5 \%$ Nonidet P40, Roche complete EDTA acid-free protease inhibitor cocktail) for $20 \mathrm{~min}$ at $4{ }^{\circ} \mathrm{C}$. Lysates were cleared by centrifugation at $12,000 \mathrm{~g}$ for $10 \mathrm{~min}$ and the resulting material subjected to IP with each antibody overnight at $4{ }^{\circ} \mathrm{C}$ with gentle inversion. Resin containing immune complexes was washed eight times with ice cold lysis buffer and followed by three times Tris-buffered saline (TBS) washes. SDS loading buffer was then added and proteins were eluted with by boiling at $95^{\circ} \mathrm{C}$ for $5 \mathrm{~min}$.

\section{Cell growth and colony formation analysis}

SMMC-7721 cells expressing Flag-control, Flag-Dlg5 WT or Flag-Dlg5 S730A. were seeded into six-well plates at $1 \times 10^{4} /$ well. Cell numbers were counted by trypan blue staining. For colony formation assays, cells were seeded in a six-well plate at a density of 1000/well and then cultured for 2 weeks. The numbers of colonies containing more than 50 cells were counted by crystal purple staining. 


\section{Xenograft assays}

Animal study was approved by Animal Care and Use Committee of the First People's of Hospital of Jingmen City. Twenty 8-week-old male nude mice were used in this study. All mice were kept in a specific pathogen-free facility. Cells at a density of $5 \times 10^{6}$ were suspended in $50 \mu \mathrm{l}$ of DMEM medium, mixed with Matrigel (Corning; $1: 1)$ and injected into the flanks of male nude mice. Tumor sizes were measured by a caliper. Tumor volumes were calculated using the formula length $\times$ width $2 \times 1 / 2$. Tumor weights were measured after mice were sacrificed, 3 weeks after injection.

\section{Statistical analyses}

Statistical analysis was performed with GraphPad Prism 7.0 software. Differences between two groups were assessed by Student's t-test. P values of $<0.05$ were considered statistically significant. Statistical significance is displayed as * $P<0.05$, ** $P<0.01$, or *** $P<0.001$.

\section{Results}

Dlg5 is regulated by the ubiquitin proteasome system via an SCF ubiquitin ligase complex

We found that the protein level of Dlg5 was regulated by the ubiquitin proteasome system, as proteasome inhibitor MG132 treatment resulted in the accumulation of endogenous Dlg5 in two HCC cell lines and exogenous expressed Flag-Dlg5 in 293T cells (Fig. 1a-c). As the Cullin-based ubiquitin E3 ligases (CRLs) are usually involved in the degradation of many key proteins during tumorigenesis [13], we then asked whether one of CRLs might be required for the degradation of Dlg5. The activity of Cullins requires NEDD8 conjugation and an investigational NEDD8-activating enzyme inhibitor MLN4924 could prevent NEDD8 modification and inactivate CRLs $[14,15]$. To this end, SMMC-7721 cells were treated with MLN4924, and we found that MLN4924 treatment significantly induced the expression of $\operatorname{Dlg} 5$ and $\mathrm{p} 27$, the latter is a well-known CRL substrate (Fig. 1d). To further test which Cullin is responsible for the degradation of Dlg5, five dominant negative (DN) Cullin members, including Cullin1, Cullin2, Cullin3, Cullin4A and Cullin4B, were expressed into $293 \mathrm{~T}$ cells, respectively. As shown in Fig. 1e, among five DN-Cullin members, only DN-Cullin1 could significantly induce the expression of Dlg5. Furthermore, silencing the expression of Cullin1 by siRNAs also resulted in the increase of Dlg5 protein (Fig. 1f), suggesting Dlg5 maybe a substrate of the SCF E3 ligase. To test this possibility, 293T cell were transfected with Flag-Dlg5 or Flag-Con for $36 \mathrm{~h}$ and Flag-Dlg5 protein complex was immunoprecipitated by Flag M2 beads and subjected to immunoblot detection. We found that both Cullin1 and Skp1 proteins were presented in the precipitated Flag-Dlg5 complex (Fig. 1g). Together, these

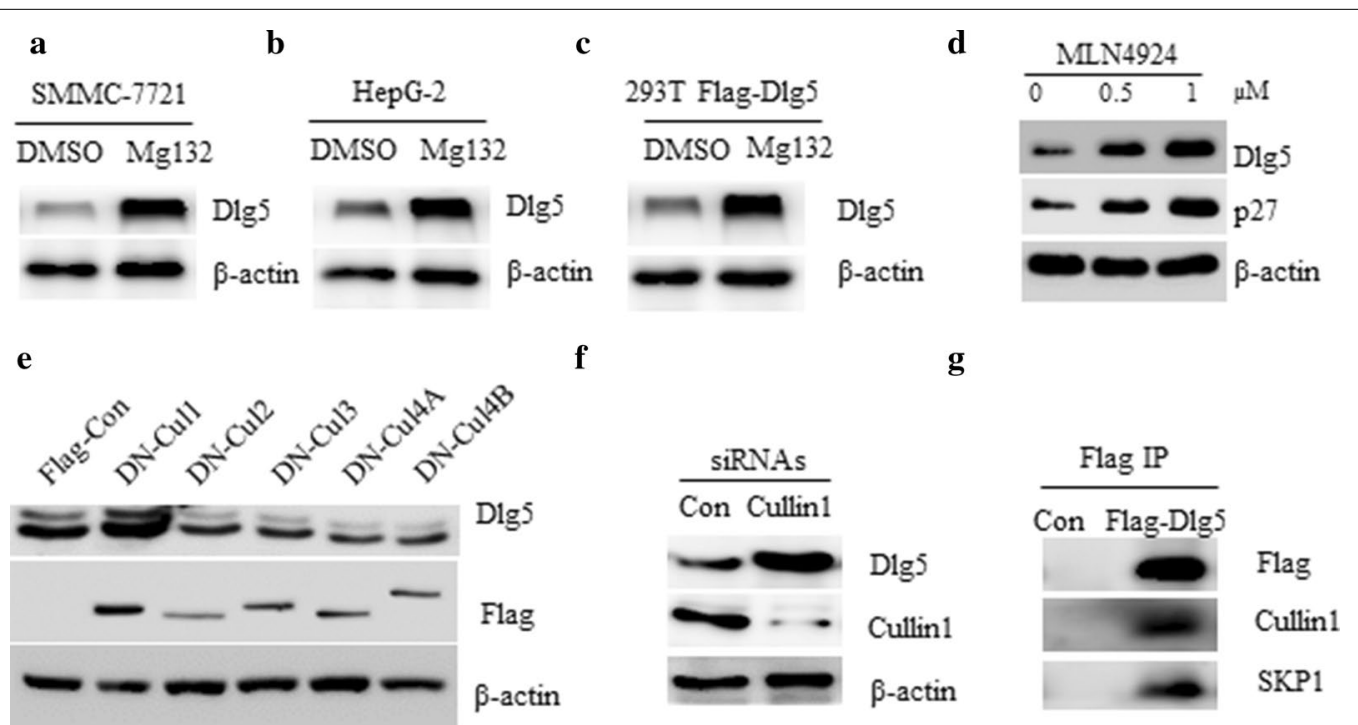

Fig. 1 Dlg5 is regulated by the ubiquitin proteasome system via an SCF ubiquitin ligase complex. a Western blot analysis of SMMC-7721 cells treated with DMSO or $10 \mu \mathrm{M} \mathrm{MG} 132$ for $4 \mathrm{~h}$. b Western blot analysis of HepG-2 cells treated with DMSO or $10 \mu \mathrm{M} \mathrm{MG} 132$ for $4 \mathrm{~h}$. c $293 \mathrm{~T}$ cells were transfected with Flag-Con or Flag-Dlg5 for $36 \mathrm{~h}$, cells were then treated with $10 \mu \mathrm{M} \mathrm{MG} 132$ for $4 \mathrm{~h}$ and subjected to western blot analysis with indicated antibodies. d Western blot analysis of 293T cells treated with indicated doses of MLN4924 for $4 \mathrm{~h}$. e Western blot analysis of $293 \mathrm{~T}$ cells transfected with DN-Cullin1, DN-Cullin2, DN-Cullin3, DN-Cullin4A or DN-Cullin4B plasmid, respectively. f Western blot analysis of SMMC-7721 cells transfected with siRNAs against control or Cullin1. g 293T cells were transfected with Flag-Con or Flag-Dlg5 for 36 h, cell lysate was subjected to immunoprecipitation by FlagM2 antibody. Immunoprecipitates were detected by western blot using indicated antibodies 
results indicate that Dlg5 is regulated by the ubiquitin proteasome system by an SCF ubiquitin ligase complex.

\section{Dlg5 is associated with $\beta-\operatorname{TrCP}$}

To better understand how Dlg5 is regulated, we searched the interacting proteins of Dlg5 through the BioGRID database (https://thebiogrid.org/). A total of 35 proteins have been reported to interact with Dlg 5 curated by both high throughput and low throughput. Interestingly, we found that the $\beta-\operatorname{TrCP}$, a substrate recruiting subunit of SCF complex, is listed as interacting protein of Dlg5 by high throughput (Fig. 2a). To confirm the interaction between $\beta$-TrCP and Dlg5, 293T cell were transfected with Flag-Dlg5 and HA- $\beta$ - TrCP for $36 \mathrm{~h}$. Flag-Dlg 5 protein complex was immunoprecipitated by Flag M2 beads and subjected to immunoblot with anti-Flag or anti-HA antibodies, respectively. As expected, HA- $\beta$ - $\operatorname{TrCP}$ was readily detected in Flag-Dlg5 immunoprecipitate (Fig. 2b). Moreover, the interaction between endogenous $\beta$-TrCP and Dlg5 was further confirmed in SMMC-7721 cells (Fig. 2c). Together, these data indicate that $\mathrm{Dlg} 5$ is associated with $\beta$-TrCP protein.

\section{$\beta-\operatorname{TrCP}$ regulates the stability and ubiquitination of Dlg5}

To investigate whether $\beta$-TrCP can regulate $\operatorname{Dlg} 5$ protein, we overexpressed $\beta$-TrCP into SMMC-7721 cells and found that overexpression of $\beta-\operatorname{TrCP}$ resulted in a marked reduction of Dlg5 levels in a dose dependent manner (Fig. 3a). Next, we used siRNA to interfere with the expression of $\beta-\operatorname{TrCP}$ in SMMC-7721 cells, and found that silencing of $\beta-\operatorname{TrCP}$ resulted in the increase of Dlg 5 protein (Fig. 3b). This increase is due to the reduced degradation of Dlg5 protein, as shown by the increase in Dlg5 half-life (Fig. 3c). Consistent with these data, silencing of $\beta$-TrCP decreased the ubiquitination form of Dlg5 (Fig. 3d). Usually, $\beta$-TrCP targets substrates for ubiquitination by recognizing a phosphodegron on them [16]. We found that Dlg5 contains a conserved $\beta$-TrCP degron (DSGxxxE) (Fig. 3e). Next, we asked whether this putative degron is sufficient for $\beta$-TrCP binding. To this end, we generated a mutant in which serine 730 was mutated to alanine (S730A), and test its binding activity to $\beta$-TrCP. In contrast to Wildtype (WT) Dlg5, the interaction between Dlg5 S730A mutant and $\beta$-TrCP was greatly impaired, if not completely (Fig. 3f), suggesting the phosphorylation of serine residues within DSGxxxE is critical for $\beta$-TrCP recognition. In concert with this, the half-life of Dlg5 S730A mutant was increased when compared with Dlg5 WT (Fig. 3g) and the K48 ubiquitination form of Dlg5 S730A mutant was decreased (Fig. 3h). Together, these data suggested that $\beta$-TrCP regulated the stability and ubiquitination of Dlg5 in a phosphorylation-dependent manner.

\section{Failure to degrade Dlg5 significantly inhibited HCC cells proliferation}

To investigate whether failure to degrade Dlg5 affects HCC cells proliferation, we analyzed SMMC-7721 cells

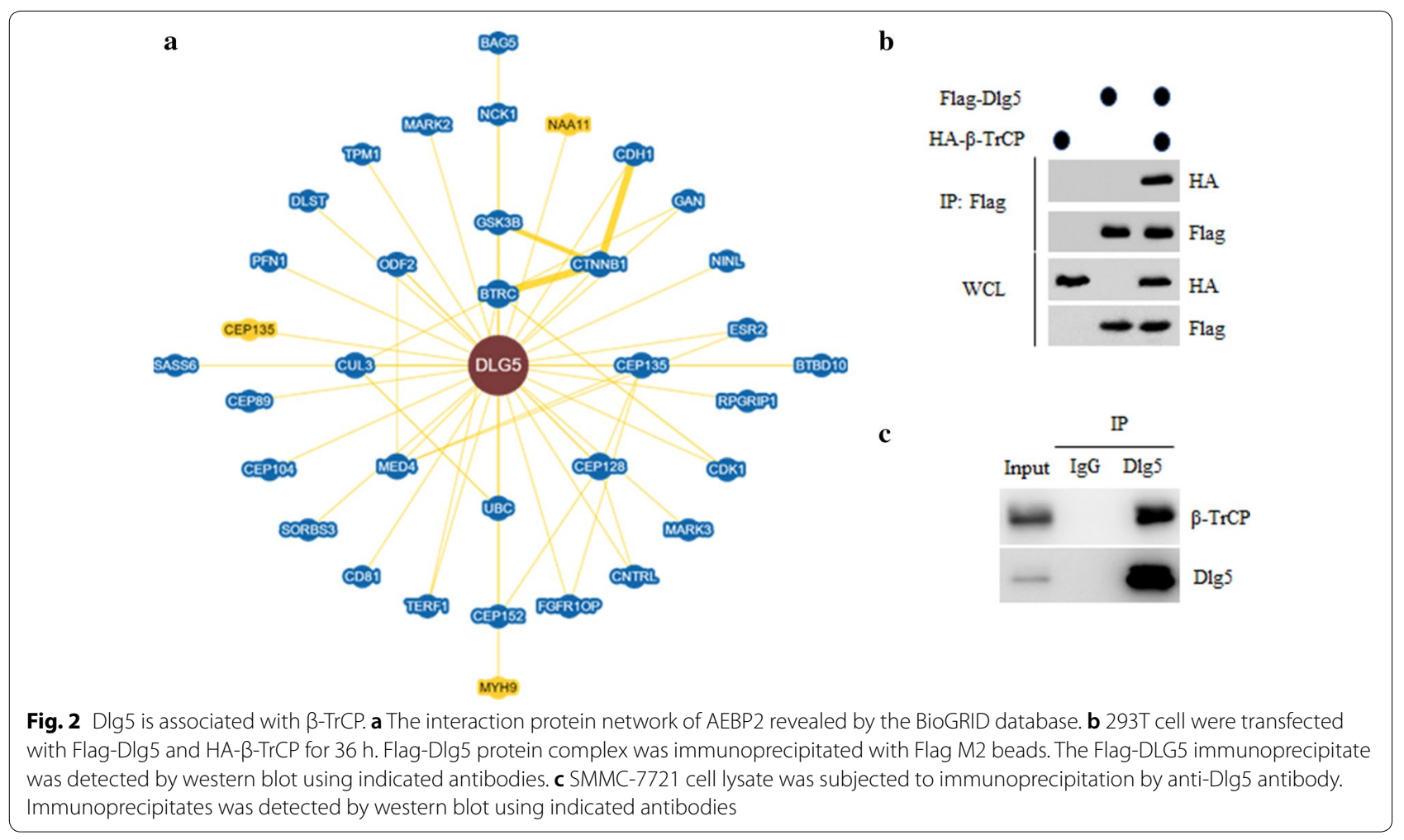




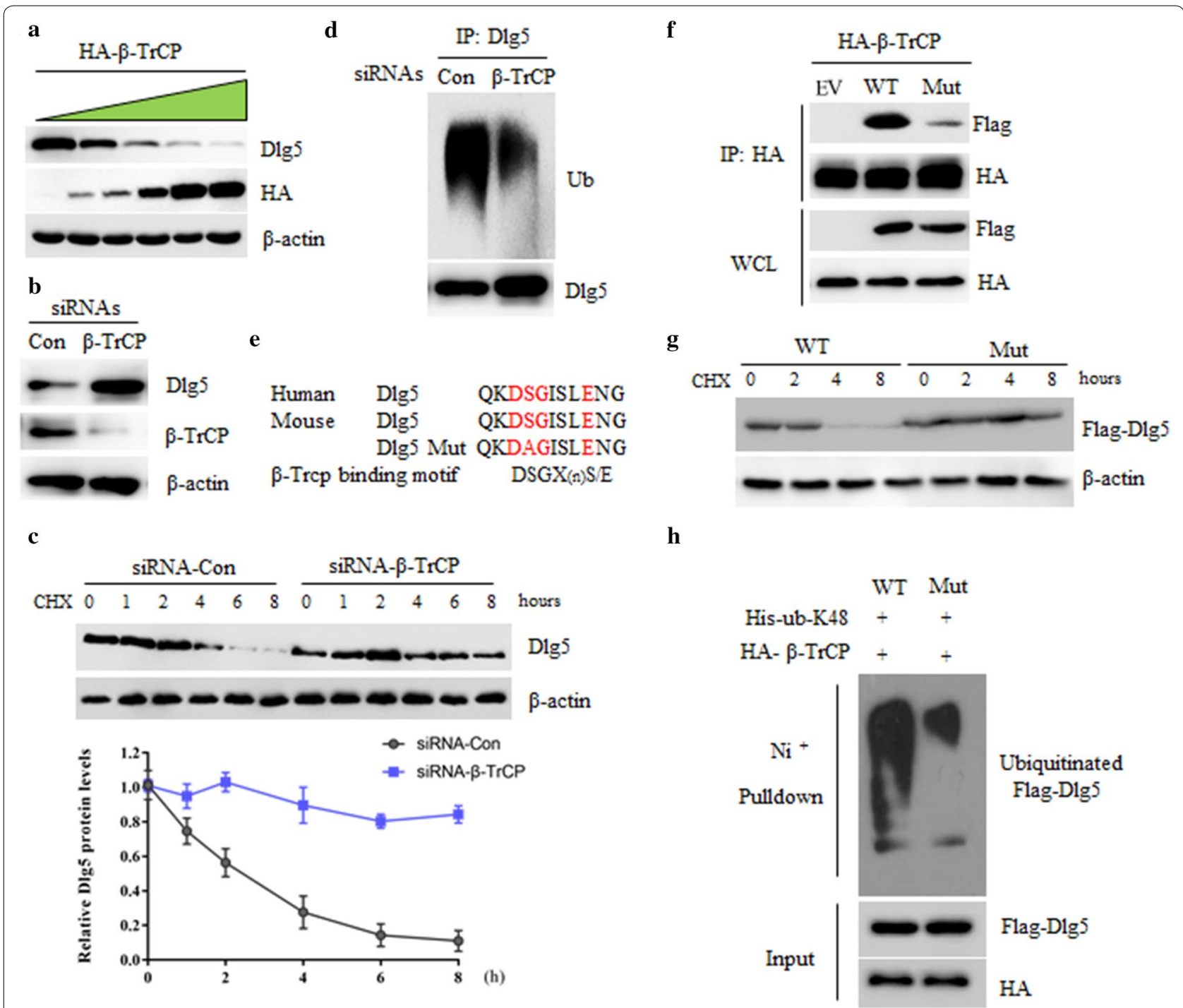

Fig. $3 \beta$-TrCP regulates the stability and ubiquitination of Dlg5. a Western blot analysis of SMMC-7721 cells transfected with indicated doses of Flag- $\beta$-TrCP. $\mathbf{b}$ Western blot analysis of SMMC-7721 cells transfected with siRNAs against control or $\beta$-TrCP. c SMMC-7721 cells transfected with siRNAs against control or $\beta-\operatorname{TrCP}$ for $24 \mathrm{~h}$, then $20 \mathrm{\mu g} / \mathrm{ml}$ cycloheximide (CHX) was added for the indicated time course. Cell extracts were subjected to western blot with the indicated antibodies. d SMMC-7721 cells transfected with siRNAs against control or $\beta$-TrCP for 36 h, cell lysate was subjected to immunoprecipitation by anti-Dlg5 antibody. Immunoprecipitates was detected by western blot using indicated antibodies. e Alignment of amino acids corresponding to the DSGxxxE sequence with Dlg5 orthologs. $f$ 293T cells were co-transfected Flag- $\beta$ - $\operatorname{TrCP}$ with HA-Dlg5 WT or HA-Dlg5 S730A for 36 h, cell lysate was subjected to immunoprecipitation by anti-Flag antibody. Immunoprecipitates was detected by western blot using indicated antibodies. $\mathbf{g} 293 \mathrm{~T}$ cells transfected with indicated plasmids for $36 \mathrm{~h}$, cell lysate was subjected to western blot detection. $\mathbf{h} 293$ T cells were co-transfected Flag- $\beta-T r C P$ and his-K48-ub with HA-Dlg5 WT or HA-Dlg5 S730A for 36 h, cell lysate was subjected to immunoprecipitation by $\mathrm{Ni}+$ purification. Immunoprecipitate was detected by western blot using indicated antibodies

expressing Flag-tagged Dlg5 WT or Flag-tagged Dlg5 S730A. As expected, overexpression of Dlg5 WT did not affect SMMC-7721 cells proliferation, however cells expressing Flag-tagged Dlg5 S730A showed decreased proliferation activity by both cell number counting and CCK-8 assays (Fig. 4a, b). Moreover, cells expressing Flag-tagged Dlg5 S730A exhibited significant deceased colony formation ability when compared with either control cells or cells expressing Dlg5 WT (Fig. 4c). We also used nude mice model to investigate whether failure to degrade Dlg5 affected cells proliferation in vivo. Four weeks old BALB/c nude mice were subcutaneously injected with $5 \times 10^{6}$ SMMC-7721 cells either expressing Flag-tagged Dlg5 WT or Flag-tagged Dlg5 S730A. As expected, SMMC-7721 cells stable expressing of a nondegradable Dlg5 S730A mutant dramatically decreased 


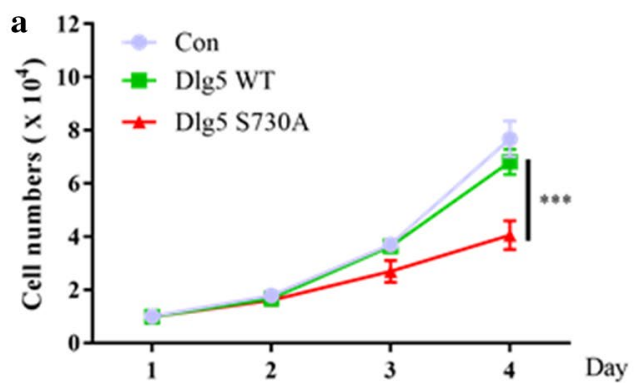

b

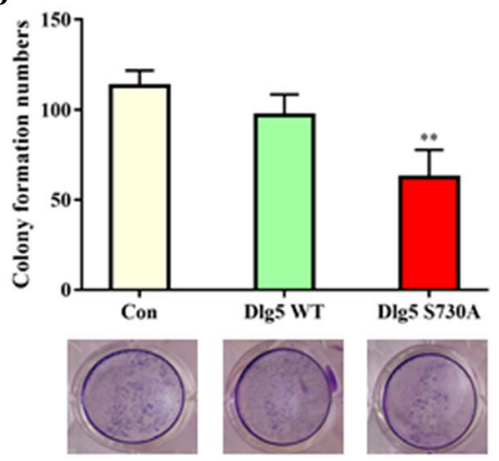

c

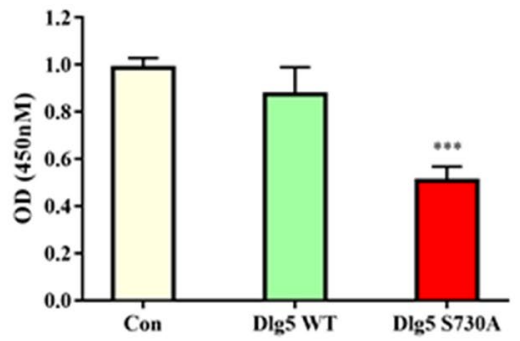

d

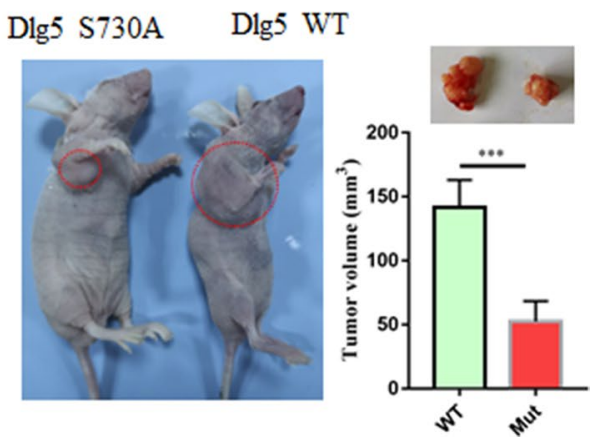

Fig. 4 Failure to degrade Dlg5 significantly inhibited HCC cells proliferation. a The cell growth curves of control, Dlg5 WT or Dlg5 S730A cells for 4 days. $\mathbf{b}$ The cell viability of control, Dlg5 WT or Dlg5 S730A cells were measured by CCK-8 assay. Data represents mean \pm SD. Results were averaged from three independent experiments. c Clone formation of control, Dlg5 WT or Dlg5 S730A cells were determined by soft agar colony formation assay. 1000 cells were seeded into each well, and cultured for another 2 weeks. Cells were stained with crystal violet and then counted. $\mathbf{d}$ Each nude mouse was subcutaneously injected with $5 \times 10^{6}$ cells stably expressing Dlg5 WT or Dlg5 S730A for 3 weeks. $n=10$ for nude mice injected with Dlg5 WT or Dlg5 S730A cells, separately

tumorigenesis, as the tumor volume was significantly decreased compared with Dlg5 WT group (Fig. 4d). Together, these data suggested that failure to degrade Dlg5 significantly inhibited HCC cells proliferation both in vitro and in vivo.

\section{Discussion}

Dlg5 is an important protein that maintains the polarity of epithelial cells and is essential for the maintenance of adhesion junctions and the formation of epithelial polarity [17]. Dlg5 knockout mice exhibited cerebral aqueduct occlusion, severe brain edema, renal cysts, emphysemalike lesions, and half of these mice were dead during perinatal [18]. The main cause of these lesions is the loss of cell adhesion junctions and epithelial polarity. Cadherin family is critical for cell adhesion junctions and epithelial polarity maintenance [19]. Dlg5 is involved in the correct localization of $\mathrm{N}$-cadherin to the cell membrane [20]. Deletion of Dlg5 will result in the failure of N-cadherin localization, which in turn destroys the adhesion junction between cells, ultimately leading to the failure of epithelial cell polarity maintenance [20].
The loss of polar proteins leads to the failure of epithelial cell polarity maintenance and is closely related to tumorigenesis [21]. The down-regulation of Dlg5 has been implicated in the malignancy of breast, prostate, bladder cancers and HCC [12, 22-24]. It has been reported that lower Dlg5 expression is correlated with advanced stages of HCC [12]. Thus, to understand how Dlg5 is regulated might shed some light on the development of HCC. It has been shown that the methylation of the promoter region of DLG5 may be associated with its low expression in some tumor tissues [23]. Here, our data reveal a previous unknown molecular mechanism for the posttranscriptional regulation of Dlg5 protein in $\mathrm{HCC}$ cells.

Firstly, we have discovered that Dlg5 undergoes sustained ubiquitination degradation in cells, and this process requires a functional SCF E3 ligase. By searching a large protein-protein interaction database, we found that Dlg5 is a $\beta$-TrCP interacting protein. Through several biochemical analysis, we verified the binding of $\beta-\operatorname{TrCP}$ to Dlg5 and found that $\beta$-TrCP promoted the proteasome-dependent degradation of Dlg5. Importantly, we found that the phosphorylation of S730 is required 
for Dlg5 ubiquitination and degradation. Dlg5 S730A mutant, which cannot be recognized by $\beta$ - TrCP, the protein stability of which was significantly enhanced, and the ubiquitination was reduced. It has been reported that overexpression of Dlg5 does not significantly affect the growth of HCC SK-Hep1 cells [12], and we recaptured this phenomenon in SMMC-7721 cells overexpressing Dlg5 WT. However, we found that overexpression of Dlg5 S730A significantly inhibited the growth of SMMC-7721 cells. The discrepancy could be explained by the different protein stability between Dlg5 WT and Dlg5 S730A. We believe that Dlg5 WT is still regulated by $\beta$-TrCPmediated ubiquitination and degradation, while Dlg5 S730A is not, and therefore enhanced its ability to inhibit cell proliferation. Consistent with these in vitro data, we also found that cells stably expressing Dlg5 S730A significantly inhibited tumor growth in nude mice than Dlg5 WT. Given the critical role of Dlg5 to maintain cell polarity, cells stably expressing Dlg5 S730A mutations will continue to maintain cell polarity and inhibit tumor development.

\section{Conclusion}

Our study is the first to show that Dlg5 is precisely regulated by different posttranscriptional ways and phosphorylation of Dlg5 at S730A is required for its ubiquitination and degradation. Our finding further provides a novel molecular mechanism for the negative regulation of Dlg 5 by $\beta$-TRCP in HCC cells. Interference with this regulatory effect on Dlg5 significantly inhibited HCC cells proliferation. Thus, our data suggest that preventing Dlg5 degradation could be a possible novel strategy for clinical treatment of $\mathrm{HCC}$.

\section{Abbreviations}

CRLs: Cullin-based ubiquitin E3 ligases; Dlg5: discs large homolog 5; HCC: hepatocellular carcinoma; IP: immunoprecipitation; MAGUK: membraneassociated guanylate kinase; SCF: Skp1/Cullin1/F-box protein; UPP: ubiquitin proteasome pathway.

\section{Acknowledgements}

Not applicable.

\section{Authors' contributions}

DW, QZ and FL participated in the design of the study and performed the experiments. DW and QZ performed the primary experience. FL and CW performed the statistical analyses. CY and HY were major contributors to the design of this study and wrote the manuscript. All authors read and approved the final manuscript.

\section{Funding}

This project is funded by the First People's of Hospital of Jingmen City.

\section{Availability of data and materials}

Please contact corresponding author for data requests.
Ethics approval and consent to participate

This study was approved by Animal Care and Use Committee of the First People's of Hospital of Jingmen City.

\section{Consent for publication}

All listed authors have actively participated in the study and have read and approved the submitted manuscript.

\section{Competing interests}

The authors declare that they have no competing interests.

\section{Author details}

${ }^{1}$ Department of Anesthesiology, The First People's of Hospital of Jingmen City, Jingmen, Hubei 448000, China. ${ }^{2}$ Department of Operation Room, The First People's of Hospital of Jingmen City, Jingmen, Hubei 448000, China. ${ }^{3}$ Department of Nursing, The First People's of Hospital of Jingmen City, Jingmen, Hubei 448000, China. ${ }^{4}$ Department of Neonatology, The First People's of Hospital of Jingmen City, Jingmen, Hubei 448000, China.

Received: 14 September 2019 Accepted: 11 November 2019

Published online: 15 November 2019

\section{References}

1. Marquardt JU, Andersen JB, Thorgeirsson SS. Functional and genetic deconstruction of the cellular origin in liver cancer. Nat Rev Cancer. 2015;15(11):653-67.

2. Sia D, Villanueva A, Friedman SL, Llovet JM. Liver cancer cell of origin, molecular class, and effects on patient prognosis. Gastroenterology. 2017:152(4):745-61

3. Yu LX, Schwabe RF. The gut microbiome and liver cancer: mechanisms and clinical translation. Nat Rev Gastroenterol Hepatol. 2017;14(9):527-39.

4. Bhattacharyya S, Yu H, Mim C, Matouschek A. Regulated protein turnover: snapshots of the proteasome in action. Nat Rev Mol Cell Biol. 2014;15(2):122-33.

5. Ciechanover A. The unravelling of the ubiquitin system. Nat Rev Mol Cell Biol. 2015;16(5):322-4.

6. Rape M. Ubiquitylation at the crossroads of development and disease. Nat Rev Mol Cell Biol. 2018;19(1):59-70.

7. Skaar JR, Pagan JK, Pagano M. SCF ubiquitin ligase-targeted therapies. Nat Rev Drug Discov. 2014;13(12):889-903.

8. Skaar JR, Pagan JK, Pagano M. Mechanisms and function of substrate recruitment by F-box proteins. Nat Rev Mol Cell Biol. 2013;14(6):369-81.

9. Jin J, Cardozo T, Lovering RC, Elledge SJ, Pagano M, Harper JW. Systematic analysis and nomenclature of mammalian F-box proteins. Genes Dev. 2004;18(21):2573-80.

10. Zheng N, Wang Z, Wei W. Ubiquitination-mediated degradation of cell cycle-related proteins by F-box proteins. Int J Biochem Cell Biol. 2016;73:99-110.

11. Liu J, Li J, Ren Y, Liu P. DLG5 in cell polarity maintenance and cancer development. Int J Biol Sci. 2014;10(5):543-9.

12. Ke Y, Bao T, Zhou Q, Wang Y, Ge J, Fu B, Wu X, Tang H, Shi Z, Lei X, et al. Discs large homolog 5 decreases formation and function of invadopodia in human hepatocellular carcinoma via Girdin and Tks5. Int J Cancer. 2017;141(2):364-76

13. Biedermann S, Hellmann H. WD40 and CUL4-based E3 ligases: lubricating all aspects of life. Trends Plant Sci. 2011;16(1):38-46.

14. Soucy TA, Smith PG, Milhollen MA, Berger AJ, Gavin JM, Adhikari S, Brownell JE, Burke KE, Cardin DP, Critchley S, et al. An inhibitor of NEDD8-activating enzyme as a new approach to treat cancer. Nature. 2009;458(7239):732-6.

15. Soucy TA, Smith PG, Rolfe M. Targeting NEDD8-activated cullin-RING ligases for the treatment of cancer. Clin Cancer Res. 2009;15(12):3912-6.

16. Frescas D, Pagano M. Deregulated proteolysis by the F-box proteins SKP2 and beta-TrCP: tipping the scales of cancer. Nat Rev Cancer. 2008;8(6):438-49.

17. Friedrichs F, Stoll M. Role of discs large homolog 5. World J Gastroenterol. 2006;12(23):3651-6. 
18. NechiporukT, Fernandez TE, Vasioukhin V. Failure of epithelial tube maintenance causes hydrocephalus and renal cysts in Dlg5-/- mice. Dev Cell. 2007;13(3):338-50.

19. van Roy F. Beyond E-cadherin: roles of other cadherin superfamily members in cancer. Nat Rev Cancer. 2014;14(2):121-34.

20. Wang SH, Celic I, Choi SY, Riccomagno M, Wang Q, Sun LO, Mitchell SP, Vasioukhin V, Huganir RL, Kolodkin AL. Dlg5 regulates dendritic spine formation and synaptogenesis by controlling subcellular $\mathrm{N}$-cadherin localization. J Neurosci. 2014;34(38):12745-61.

21. Ellenbroek SI, Iden S, Collard JG. Cell polarity proteins and cancer. Semin Cancer Biol. 2012;22(3):208-15.

22. Liu J, Li J, Li P, Wang Y, Liang Z, Jiang Y, Li J, Feng C, Wang R, Chen H, et al. Loss of DLG5 promotes breast cancer malignancy by inhibiting the Hippo signaling pathway. Sci Rep. 2017;7:42125.
23. Zhou Z, Guo Y, Liu Y, Zhang F, Wang Y, Shen B, Qin Y, Qiu J. Methylationmediated silencing of Dlg5 facilitates bladder cancer metastasis. Exp Cell Res. 2015;331(2):399-407.

24. Tomiyama L, Sezaki T, Matsuo M, Ueda K, Kioka N. Loss of Dlg5 expression promotes the migration and invasion of prostate cancer cells via Girdin phosphorylation. Oncogene. 2015;34(9):1141-9.

\section{Publisher's Note}

Springer Nature remains neutral with regard to jurisdictional claims in published maps and institutional affiliations.
Ready to submit your research? Choose BMC and benefit from:

- fast, convenient online submission

- thorough peer review by experienced researchers in your field

- rapid publication on acceptance

- support for research data, including large and complex data types

- gold Open Access which fosters wider collaboration and increased citations

- maximum visibility for your research: over $100 \mathrm{M}$ website views per year

At BMC, research is always in progress.

Learn more biomedcentral.com/submissions 